\title{
Submolecular Resolution by Variation of the Inelastic Electron Tunneling Spectroscopy Amplitude and its Relation to the AFM/STM Signal
}

\author{
Bruno de la Torre, ${ }^{1,2, *}$ Martin Švec, ${ }^{1,2}$ Giuseppe Foti, ${ }^{1}$ Ondřej Krejčí $1,{ }^{1,3}$ Prokop Hapala, ${ }^{1}$ Aran Garcia-Lekue,,${ }^{4,5}$ \\ Thomas Frederiksen, ${ }^{4,5}$ Radek Zbořil, ${ }^{2}$ Andres Arnau, ${ }^{4}$ Héctor Vázquez, ${ }^{1}$ and Pavel Jelínek ${ }^{1,2,4, \dagger}$ \\ ${ }^{1}$ Institute of Physics, Academy of Sciences of the Czech Republic, v.v.i., Cukrovarnická 10, 162 O0 Prague, Czech Republic \\ ${ }^{2}$ Regional Centre of Advanced Technologies and Materials, Palacký University, Šlechtitelì 27, 78371 Olomouc, Czech Republic \\ ${ }^{3}$ Faculty of Mathematics and Physics, Department of Surface and Plasma Science, Charles University, \\ $V$ Holešovičkách 2, 18000 Prague, Czech Republic \\ ${ }^{4}$ Donostia International Physics Center (DIPC), Paseo Manuel Lardizabal 4, E-20018 San Sebastian, Spain \\ ${ }^{5}$ Ikerbasque, Basque Foundation for Science, 48013 Bilbao, Spain
}

(Received 29 June 2017; revised manuscript received 11 August 2017; published 16 October 2017)

\begin{abstract}
Here we show scanning tunneling microscopy (STM), noncontact atomic force microscopy (AFM), and inelastic electron tunneling spectroscopy (IETS) measurements on an organic molecule with a CO-terminated tip at $5 \mathrm{~K}$. The high-resolution contrast observed simultaneously in all channels unambiguously demonstrates the common imaging mechanism in STM/AFM/IETS, related to the lateral bending of the CO-functionalized tip. The IETS spectroscopy reveals that the submolecular contrast at $5 \mathrm{~K}$ consists of both renormalization of vibrational frequency and variation of the amplitude of the IETS signal. This finding is also corroborated by first principles simulations. We extend accordingly the probe-particle AFM/STM/IETS model to include these two main ingredients necessary to reproduce the high-resolution IETS contrast. We also employ the first principles simulations to get more insight into a different response of frustrated translation and rotational modes of the $\mathrm{CO}$ tip during imaging.
\end{abstract}

DOI: 10.1103/PhysRevLett.119.166001

The development of high-resolution scanning tunneling microscopy (STM) [1], atomic force microscopy (AFM) [2], and inelastic electron tunneling spectroscopy (IETS) [3] imaging with functionalized tips has allowed us to reach unprecedented spatial resolution of organic molecules on surfaces. Using these techniques, the chemical structure of molecules can be now routinely determined directly from experimental images [4,5], as well as the information about bond order [6], intermediates, and products of onsurface chemical reactions [7] or charge distribution within molecules [8].

The origin of the high-resolution AFM/STM imaging is now well understood within the framework of the so-called probe-particle (PP) model [9-11]. In general, an atom or molecule (the probe particle) placed at the tip apex is sensitive to spatial variations of the potential energy landscape of the molecule resulting from the interplay between Pauli, electrostatic, and van der Waals interactions $[9,10,12,13]$. At close tip-sample distances, the probe particle relaxes according to the potential energy surface, which gives rise to a sharp submolecular contrast. Nevertheless, neither direct experimental evidence nor a unified description of the imaging mechanism for all three scanning modes has been presented.

On one hand, noncontact AFM is most often used in the high-resolution imaging mode, which provides the highest spatial resolution with relatively straightforward interpretation, compared to the other two modes. This implies that the instrument operates in the frequency modulation (FM) mode
[14], which is not a trivial task from both the instrumental and data acquisition point of view. From this perspective, the STM mode seems to be a more feasible choice. On the other hand, the interpretation of high-resolution STM images is not at all straightforward because of the convolution of the geometric and electronic effects [11]. The IETS mode [15] thus represents a promising alternative [3], but the highresolution contrast was so far only demonstrated at subKelvin temperatures. This temperature requirement poses severe limitations for its wider application.

In this Letter, we present simultaneous AFM/STM/IETS measurements of iron(II) phtalocyanine (FePc) on $\mathrm{Au}(111)$ acquired with a CO-terminated tip at $5 \mathrm{~K}$ [16]. These measurements (i) demonstrate that high-resolution IETS imaging is also feasible with standard LHe bath cryostats, and (ii) experimentally confirm the common imaging mechanism for all three imaging modes. Atomistic simulations using nonequilibrium Green's functions (NEGF) [27] and an extension of the PP model $[3,10,16]$ provide a characterization of the contrast in the inelastic signal and a unified description of AFM/STM/IETS.

Figure 1(a) shows a constant-current STM image of coadsorbed $\mathrm{FePc}$ and $\mathrm{CO}$ molecules on $\mathrm{Au}(111)$. Prior to the high-resolution imaging, a $\mathrm{CO}$ molecule was picked up to the tip. The presence of the $\mathrm{CO}$ on the tip is confirmed by the characteristic low-energy IETS spectrum over the bare substrate, consisting of the frustrated translational (FT) and frustrated rotational $(\mathrm{FR})$ modes located at $\approx 3$ and 

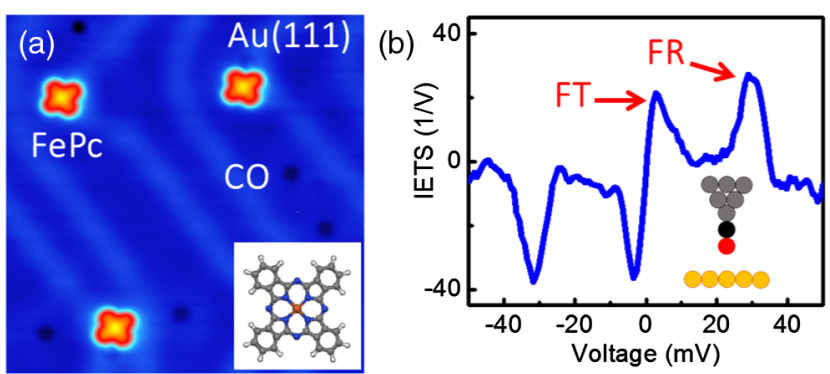

FIG. 1. Constant current STM images of FePc on $\mathrm{Au}(111)$ and IETS signal of a CO-functionalized tip. (a) $16 \times 16 \mathrm{~nm}^{2}$ constant-current STM image of the $\mathrm{Au}(111)$ surface and the coadsorbed $\mathrm{CO}$ and $\mathrm{FePc}$ molecules, acquired at the $-200 \mathrm{mV}$ and $10 \mathrm{pA}$ set point. (b) STM-IETS of the CO adsorbed on the tip apex taken at the bare Au surface, showing the vibrational modes. The FR and FT modes of the $\mathrm{CO}$ are resolved. Bias voltage modulation was $3 \mathrm{mV}$ at $963 \mathrm{~Hz}$ frequency. Stabilization set point $V=50.0 \mathrm{mV}$ and $I=3.0 \mathrm{nA}$.

$\approx 30 \mathrm{meV}$, respectively, as shown in Fig. 1(b). These values are similar to the previous IETS spectra of a CO molecule adsorbed on $\mathrm{Ag}(110)$ or $\mathrm{Cu}(111)[3,28,29]$. In repeated attempts, we noticed that the amplitude and the shape of the FT peak in the IETS spectrum of the CO tip are very sensitive to the configuration of the metal tip apex before its functionalization [28]. For this reason, the metal apex preparation and $\mathrm{CO}$ picking process were repeated until an intense and regular IETS spectrum was obtained.

In Fig. 2(a) we present the maps of signals obtained while scanning with the CO-terminated tip in three constant heights above the molecule. These are the tunneling current $I_{t}$, frequency shift $\Delta f, d^{2} I / d V^{2}$, and its normalized value $\left(d^{2} I / d V^{2}\right) /(d I / d V)$, denoted in the following as STM, AFM, IETS, and norm. IETS, respectively. The bias voltage was set to $3 \mathrm{mV}$ in order to optimize the sensitivity of the IETS signal to the peak corresponding to the FT vibrational mode. The STM contrast is dominated by a strong signal in the central part of the molecule, suppressing significantly the submolecular resolution of the molecule. We attribute the strong signal in the central part to $d$ orbitals of Fe atom just below the Fermi level.

On the other hand, both AFM and IETS images exhibit sharp edges that reveal the backbone of the FePc molecule. At the closest distances the AFM contrast inverts and the contrast in the IETS map is enhanced. The $\Delta f(Z)$ spectroscopy shown in Fig. S1 [16] proves that the images were recorded at tip-sample separations where the repulsive interaction plays a dominant role. These observations are fully consistent with the assertion that the characteristic sharp edges in the AFM images are the direct consequence of the lateral bending of the $\mathrm{CO}$ due to the repulsive interaction [9]. Since the apparent positions of the sharp edges found in the IETS map correspond almost exactly to the AFM and STM images taken at the same moment, we can infer that the effect of $\mathrm{CO}$ bending indeed plays an (a)

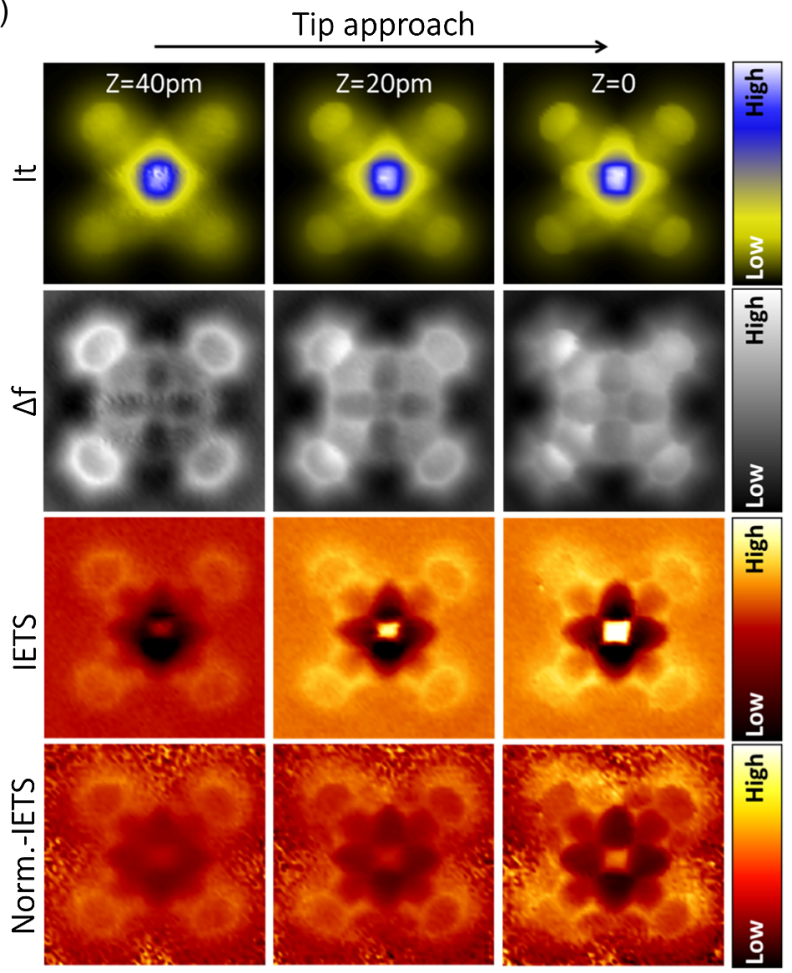

(b)

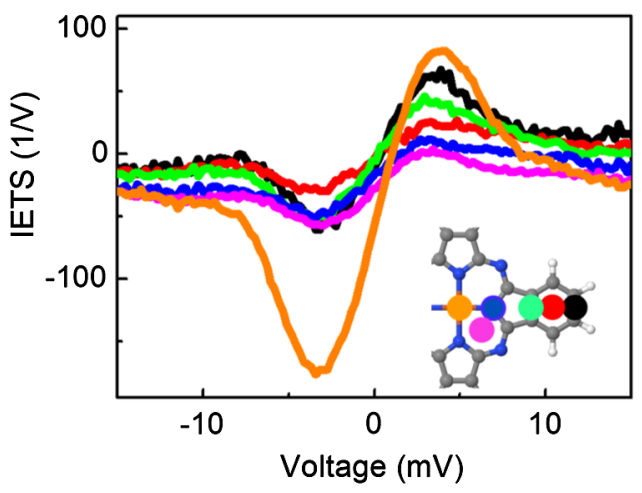

FIG. 2. Simultaneous AFM/STM/IETS images of FePc/Au(111) with a CO-functionalized tip and spatial variation of the FT mode. (a) Set of simultaneous $\left(1.6 \times 1.6 \mathrm{~nm}^{2}\right)$ current, $\Delta f$, IETS, and normalized-IETS constant-height images acquired at three different tip-sample distances with a $\mathrm{CO}$ decorated tip. The images were acquired with bias voltage of $3.0 \mathrm{mV}$. Both $\Delta f$ and IETS images show the sharp edges related with the geometric structure of the FePc molecule. (b) Spatial dependence of the frustrated translational mode for the CO-functionalized tip above the $\mathrm{FePc}$ molecule.

indispensable role in the IETS imaging as it was predicted theoretically [10].

Next, we focus on the spatial variation of the FT signal over the FePc molecule. In their seminal work, Chiang et al. [3] performed spatially resolved IETS measurements at $600 \mathrm{mK}$ above Co-phtalocyanine molecules. The high spectral resolution allowed them to resolve a subtle variation of the FT vibrational energy (frequency) and clearly showed a submolecular contrast. Later a 
theoretical explanation of the imaging mechanism was suggested, based on the frequency change of the mode [10]. However, the IETS spectra acquired for the FT mode at various locations of the FePc molecule at $5 \mathrm{~K}$, shown in the Fig. 2(b), reveal that the skeleton of FePc can be clearly resolved purely from variation of the amplitude of the IETS signal, even though variation of frequency cannot be directly observed due to broadening of the peak.

Remarkably, the highest amplitude is detected above the center of the FePc molecule, similarly to the STM $d I / d V$ images. The IETS signal is expected to scale with the magnitude of the differential conductance $d I / d V$ measured at the energy of the FT mode. Indeed, in the normalized IETS images $\left(d^{2} I / d V^{2}\right) /(d I / d V)$ the signal in the center is suppressed and the molecular structure is still clearly resolved although the normalization impairs the signalto-noise ratio in the outer regions of the molecule.

For a further understanding of the origin of the contrast observed in the IETS images, we analyze the IETS signal at two different sites over one peripheral benzene ring of the FePc molecule. The spectra were acquired at constant height using the same parameters for the lock-in as during the IETS image acquisition. Figure 3(a) shows two IETS curves, obtained on a carbon bond (black) and at the center of the benzene ring (red) for comparison. The IETS shows a strong variation of the amplitude of the FT mode while the FR remains almost constant. This observation also documents that it is not possible to clearly resolve the molecular structure using the FR mode [3].

(a)

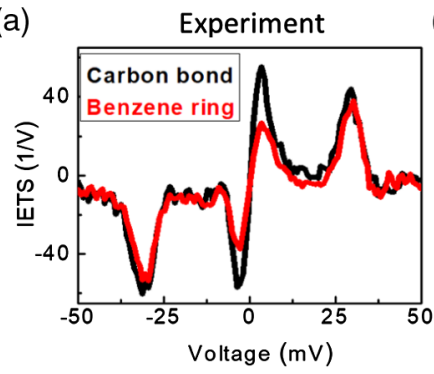

(b) NEGF Calculation

(c)

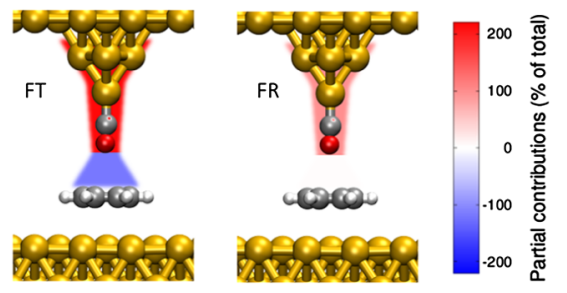

FIG. 3. Variation of IETS signal with tip position. Experimental (a) and calculated (b) IETS of the FT and FR modes above a carbon bond (black) and a benzene ring (red). Both experimental and calculated plots show the variation of the amplitude in the FT mode responsible for the IETS contrast. (c) Origin of the inelastic signal (as a fraction of the total inelastic amplitude) above the benzene ring. The signal for FR modes originates mainly in the $\mathrm{CO}$ and tip. However, for FT modes, the CO-benzene coupling also plays a significant role.
To get more insight into the imaging mechanism, we carry out first principles calculations of the inelastic signal on a simplified system consisting of a CO-functionalized tip placed above a benzene molecule. As we show below, this system is already sufficient to explain the experimental measurements. Details and methodology are given in Supplemental Material [16].

Figure 3(b) shows the calculated IETS spectrum for the CO-functionalized tip above a carbon bond and the benzene ring. Consistently with the experiments, the intensity of the calculated FT peak is reduced by a factor $\sim 2$, when going from the carbon bond to the benzene ring, while the intensity of the FR peaks is almost unchanged.

To understand why the inelastic signal of the FT modes changes strongly with tip position but not that of the FR modes, we analyze the different contributions that give rise to the inelastic peaks. The inelastic signal can be understood in terms of a Fermi golden rule involving the deformation potential and the left- and right-incident scattering states [30]. The use of a local-orbital basis enables us to group the contributions of the various regions of the junction where the inelastic signal is generated [31]. We consider a first set of contributions involving the $\mathrm{CO}$ molecule and the Au tip, and a second set consisting of the CO-benzene coupling (see [16] for details). The effect of the remaining terms is negligible. The modulus square of the sum of all contributions gives the calculated intensity of each inelastic peak.

These contributions for the most active pair of eigenchannels are shown in Fig. 3(c) for the CO-functionalized tip above the benzene ring. For clarity this is shown for only one FT and one FR mode but results are similar for all FT and FR modes on both configurations (details are given in Supplemental Material [16]). The boxes in Fig. 3(c) show the fraction of the total inelastic signal arising from each set of contributions: $\mathrm{CO}$ and tip, and the coupling of $\mathrm{CO}$ to the benzene substrate. The color scale quantifies the magnitude of each set of contributions relative to the total.

From the results shown above, we infer that there are clear differences in the origin of FT and FR modes. The FR mode is well described by the first set of contributions only. However, in order to properly capture the intensity of the FT mode, it is necessary to include these contributions as well as the off-diagonal terms between $\mathrm{CO}$ and benzene. Thus the inelastic signal of the FR mode is generated almost completely on the $\mathrm{CO}$ and tip alone, while that of the FT mode also involves the interaction with the benzene molecule.

The consequences of this localization of the inelastic signal are illustrated by constraining the dynamical region to just the $\mathrm{C}$ or $\mathrm{O}$ atom (see Supplemental Material [16]). When only the vibrations of the $\mathrm{C}$ atom are considered, the inelastic signal is unchanged with tip position. However, when only the $\mathrm{O}$ atom is allowed to vibrate, the calculated inelastic signal changes substantially from carbon bond to benzene ring positions. Thus the sensitivity of the inelastic 
peaks to the interaction with the molecular substrate rests on the composition of the vibrational modes of CO. In FR modes the larger displacement corresponds to the $\mathrm{C}$ atom, close to the tip. In FT modes, on the other hand, the displacement is larger on the $\mathrm{O}$ atom, which, being closer to the benzene molecule, is more affected by the interaction with the molecular substrate. The higher sensitivity of FT modes to the position of $\mathrm{CO}$ above the molecule follows intuitively from this result.

To describe the variations of the IETS amplitude of the FT mode, we extend the fast PP-IETS method to calculate IETS maps [10]. We carry out an approximation of the perturbative inelastic transport theory [30] and consider that the IETS signal is proportional to two terms only: (i) Variation of the tunneling hopping $T_{m n}$ between the electronic states of the tip $(m)$ and of the sample $(n)$ with respect to displacement of $\mathrm{PP}$ along vibration eigenmode $\mathbf{v}_{\lambda}$, and (ii) a prefactor depending on the energy of the vibrational mode $\omega_{\lambda}$. Intuitively the prefactor represents the magnitude of the $\mathrm{CO}$ displacement during vibration, which is prolonged as the vibration mode becomes softer due to concave potential over the bonds [10]. The full derivation of the approach can be found in Supplemental Material [16]. This approximation is justified by the NEGF analysis, which revealed dominant contribution of oxygen to the IETS signal of the FT mode [16]. Consequently, the IETS signal $\gamma_{\mathrm{FT}}$ of the FT modes is defined as follows:

$$
\gamma_{\mathrm{FT}} \sim \sum_{\lambda, m, n} C \frac{1}{\omega_{\lambda} M_{\mathrm{PP}}}\left|\frac{\partial T_{m n}}{\partial \mathbf{v}_{\lambda}}\right|^{2}
$$

where $C$ is a constant and $M_{\mathrm{PP}}$ the effective PP mass [16]. The vibrational mode $\lambda$ goes over two FT modes. This PPIETS method was implemented into the PP-code allowing simulation of the HR-STM and AFM images [9-11]. The computational cost of IETS images using this method is similar to standard STM simulation. The calculation of the high-resolution AFM/STM/IETS images relies on atomic and electronic structure of the adsorbed molecules on the surface. Therefore, we performed total energy DFT simulations of FePc molecule on an $\mathrm{Au}(111)$ surface with the FHI-Aims code [32] using the Perdew-Burke-Ernzerhof functional [33] and Tkatchenko-Scheffler vdW model [34] for geometry optimization. The electronic states used as input for PP-STM and PP-IETS codes were then calculated with the hybrid B3LYP functional [35] to provide better description of the metal-organic system.

Electronic states of the CO-functionalized tip were approximated by $p_{x}, p_{y}$, and $s$ orbitals on the probe particle to represent $\pi$ and $\sigma$ conductance channels [11]. More details about parameters of the total energy DFT and PP-SPM simulations can be found in Supplemental Material [16].

Figure 4 shows the calculated high-resolution AFM, STM, and IETS images of the FePc molecule on the $\mathrm{Au}(111)$ surface for different tip-sample distances. The

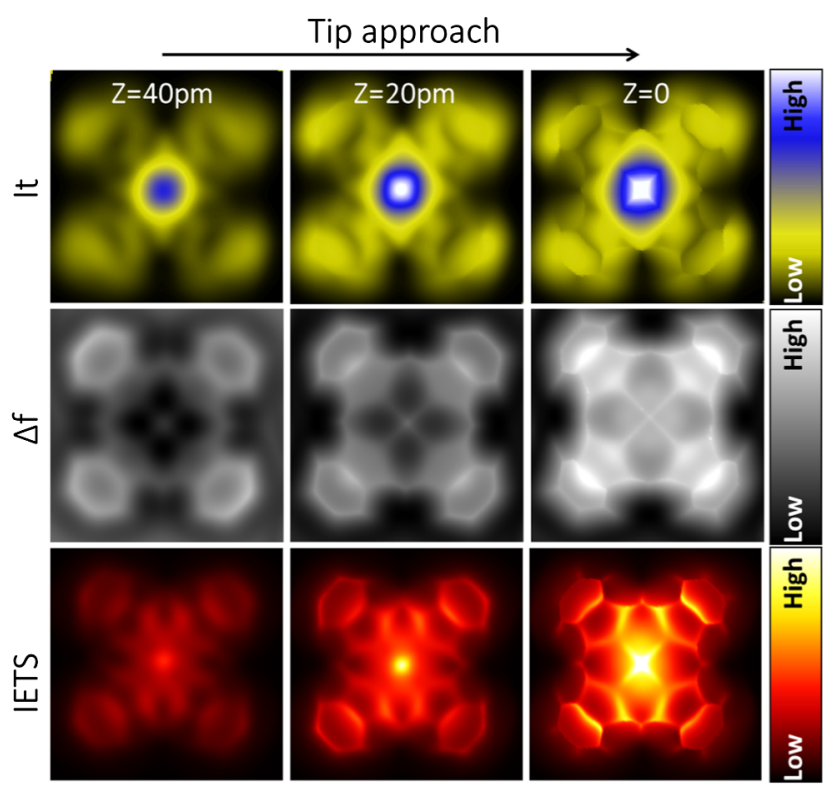

FIG. 4. Theoretical simulations of STM/AFM/IETS of FePc on the $\mathrm{Au}(111)$ surface at three different tip-sample distances. The tunneling current (top), $\Delta f$ (middle), and IETS (bottom) images were calculated using the PP-SPM model.

simulated images capture well most of the characteristic features observed experimentally in all channels. In the STM mode, we are able to reproduce the dominant contrast observed in the center of the molecule, while a sharp contrast on external benzene rings is visible only in the close distance. On the other hand, the calculated STM images contain subtle submolecular features, which are not seen in the experiment. The AFM simulation reproduces very well both the characteristic sharpening of contrast and the contrast inversion at close distances. The only discrepancy consists of a missing rectangular feature in the center of the FePc molecule. We attribute these discrepancies to a peculiar charge distribution or structural relaxation between Fe and ligand, which is not described well within DFT approximation, or possibly to some chemical force between a $\mathrm{CO}$ tip and $\mathrm{Fe}$ atom that cannot be captured using a Lennard-Jones force field. The IETS channel resolves the molecular skeleton, with the characteristic bright spot in the center of the molecule in good agreement with the experimental evidence.

From PP-IETS simulations we can also analyze the influence of two contributions on the amplitude of the IETS signal (see Fig. S13 in [16]). We found that the prefactor $1 / \omega_{\lambda}$ [Eq. (1)] is important only at close tip-sample distances due to fast decay of Pauli repulsion, which leads to concave curvature of interaction potential over the bonds. However, the molecular structure is resolved, both in the theory and experiment, over a range of $\approx 0.8 \AA$ (see Fig. S1 in [16]). The IETS contrast at far distance is mainly caused by spatial variation of inelastic tunneling matrix element, as shown both by NEGF and PP-IETS simulations. 
In conclusion, we demonstrated that the high-resolution IETS imaging with a CO-functionalized tip is feasible at $5 \mathrm{~K}$ with a spatial resolution superior to STM and comparable to AFM. Thus we believe that the IETS mode is promising for STM-only setups. The high-resolution contrast observed in all channels simultaneously demonstrates unambiguously the common imaging mechanism of the AFM/STM/IETS, related to lateral bending of the CO-functionalized tip. We provided detailed theoretical analysis of FT and FR vibrational modes showing their different localization in the STM junction. This explains why FT displays a large variation during scanning while FR is insensitive. This result opens the way to tune the surface sensitivity of the inelastic signal through appropriate molecular functionalization of the tip. We also showed that the submolecular contrast emerges not only from the changes of the $\mathrm{CO}$ vibrational frequency of the FT mode, but also due to the variation of the amplitude of the IETS signal. Finally, we extended accordingly the probeparticle AFM/STM/IETS model to include these two main ingredients necessary to reproduce the high-resolution IETS contrast.

We thank A. Peronio for fruitful discussions of the IETS signal. This research was financially supported by: the Czech Science Foundation (GACR) under Grants No. 1519672S, No. 17-24210Y, the Purkyne Fellowship and Praemium Academiae program of the Academy of Sciences of the Czech Republic, and the European Union's Horizon 2020 research and innovation program under the Marie Sklodowska-Curie Grant No. 709114. The authors also acknowledge support from Ministry of Education, Youth and Sport of the Czech Republic (NanoEnviCz, Grant No. LM2015073, and Grant No. LO1305), Grant No. FP7 FET-ICT "Planar Atomic and Molecular Scale devices" (PAMS) project (funded by the European Commission under Contract No. 610446), Spanish Ministerio de Economia y Competitividad (MINECO) (Grant No. MAT2016-78293-C6-4-R), and the Basque Government (Dep. de Educacion and UPV/ EHU, Grants No. IT-756-13 and No. PI-2016-1-0027). We thank CESNET Grant No. LM2015042 and CERIT Scientific Cloud Grant No. LM2015085, under the program "Projects of Large Research, Development, and Innovations Infrastructures" for computational resources.

*bdelatorre@fzu.cz †jelinekp@fzu.cz

[1] R. Temirov, S. Soubatch, O. Neucheva, A. Lassise, and F. Tautz, New J. Phys. 10, 053012 (2008).

[2] L. Gross, F. Mohn, N. Moll, P. Liljeroth, and G. Meyer, Science 325, 1110 (2009).

[3] C. Chiang, C. Xu, Z. Han, and W. Ho, Science 344, 885 (2014).

[4] L. Gross, N. Moll, G. Meyer, R. Ebel, W. Abdel-Mageed, and M. Jaspars, Nat. Chem. 2, 821 (2010).
[5] B. Schuler, G. Meyer, D. Pena, O. Mullins, and L. Gross, J. Am. Chem. Soc. 137, 9870 (2015).

[6] L. Gross, F. Mohn, N. Moll, B. Schuler, A. Criado, E. Guitian, D. Pena, A. Gourdon, and G. Meyer, Science 337, 1326 (2012).

[7] D. de Oteyza, P. Gorman, Y.-C. Chen, S. Wickenburg, A. Riss, D. Mowbray, G. Etkin, Z. Pedramrazi, H.-Z. Tsai, A. Rubio, M. Crommie, and F. Fischer, Science 340, 1434 (2013).

[8] P. Hapala, M. Švec, O. Stetsovych, N. van der Heijden, M. Ondracek, J. van der Lit, P. Mutombo, I. Swart, and P. Jelínek, Nat. Commun. 7, 11560 (2016).

[9] P. Hapala, G. Kichin, C. Wagner, F. S. Tautz, R. Temirov, and P. Jelínek, Phys. Rev. B 90, 085421 (2014).

[10] P. Hapala, R. Temirov, F. S. Tautz, and P. Jelínek, Phys. Rev. Lett. 113, 226101 (2014).

[11] O. Krejči, P. Hapala, M. Ondráček, and P. Jelínek, Phys. Rev. B 95, 045407 (2017).

[12] N. Moll, L. Gross, F. Mohn, A. Curioni, and G. Meyer, New J. Phys. 14, 083023 (2012).

[13] S. K. Hämäläinen, N. van der Heijden, J. van der Lit, S. den Hartog, P. Liljeroth, and I. Swart, Phys. Rev. Lett. 113, 186102 (2014).

[14] T. R. Albrecht, P. Grütter, D. Horne, and D. Rugar, J. Appl. Phys. 69, 668 (1991).

[15] B. Stipe, M. Rezaei, and W. Ho, Science 280, 1732 (1998).

[16] See Supplemental Material at http://link.aps.org/ supplemental/10.1103/PhysRevLett.119.166001 for more details, which includes Refs. [17-26].

[17] L. Bartels, G. Meyer, and K. Rieder, Appl. Phys. Lett. 71, 213 (1997).

[18] I. Horcas, R. Fernández, J. Gómez-Rodríguez, J. Colchero, J. Gómez-Herrero, and A. Baro, Rev. Sci. Instrum. 78, 013705 (2007).

[19] W. L. Jorgensen and J. Tirado-Rives, J. Am. Chem. Soc. 110, 1657 (1988).

[20] M. Brandbyge, J.-L. Mozos, P. Ordejón, J. Taylor, and K. Stokbro, Phys. Rev. B 65, 165401 (2002).

[21] M. Dion, H. Rydberg, E. Schröder, D. C. Langreth, and B. I. Lundqvist, Phys. Rev. Lett. 92, 246401 (2004).

[22] G. Foti, D. Sánchez-Portal, A. Arnau, and T. Frederiksen, Phys. Rev. B 91, 035434 (2015).

[23] G. Román-Pérez and J. M. Soler, Phys. Rev. Lett. 103, 096102 (2009).

[24] J. M. Soler, E. Artacho, J. D. Gale, A. García, J. Junquera, P. Ordejón, and D. Sánchez-Portal, J. Phys. Condens. Matter 14, 2745 (2002).

[25] J.-T. Lü, R. B. Christensen, G. Foti, T. Frederiksen, T. Gunst, and M. Brandbyge, Phys. Rev. B 89, 081405 (2014).

[26] J. Taylor, H. Guo, and J. Wang, Phys. Rev. B 63, 245407 (2001).

[27] T. Frederiksen, M. Paulsson, M. Brandbyge, and A.-P. Jauho, Phys. Rev. B 75, 205413 (2007).

[28] C. Xu, C. L. Chiang, Z. Han, and W. Ho, Phys. Rev. Lett. 116, 166101 (2016).

[29] N. Okabayashi, A. Gustafsson, A. Peronio, M. Paulsson, T. Arai, and F. J. Giessibl, Phys. Rev. B 93, 165415 (2016). 
[30] M. Paulsson, T. Frederiksen, H. Ueba, N. Lorente, and M. Brandbyge, Phys. Rev. Lett. 100, 226604 (2008).

[31] G. Foti and H. Vázquez, Phys. Rev. B 94, 045418 (2016).

[32] V. Blum, R. Gehrke, F. Hanke, P. Havu, V. Havu, X. Ren, K. Reuter, and M. Scheffler, Comput. Phys. Commun. 180, 2175 (2009).
[33] J. P. Perdew, K. Burke, and M. Ernzerhof, Phys. Rev. Lett. 77, 3865 (1996).

[34] A. Tkatchenko and M. Scheffler, Phys. Rev. Lett. 102, 073005 (2009).

[35] S. H. Vosko, L. Wilk, and M. Nusair, Can. J. Phys. 58, 1200 (1980). 